timeframes. Similar expression method was applied to hippocampi obtained from children with MTLE and normal controls. Results The expression pattern for the miR-124, miR-134 and miR-9 nearly showed the same dynamics in the three stages of MTLE development to be upregulated in the acute and chronic stages and nearly equal to the control in the latent stage, they upregulated also in the children with MTLE.

Conclusions The significant upregualtion for the brain specific miR-124, miR-134 and miR-9 in the seizures related stages and children suggested that both can be a potential targets for anticonvulsant drugs in the epileptic developing brains.

\section{PO-0850 RESTING STATE NETWORKS IN PRETERM INFANTS WITH AND WITHOUT INTRAUTERINE GROWTH RESTRICTION}

${ }^{1} \mathrm{~N}$ Padilla, ${ }^{2} \mathrm{P}$ Fransson, ${ }^{3} \mathrm{~A}$ Donaire, ${ }^{4} \mathrm{H}$ Lagercrantz, ${ }^{5} \mathrm{E}$ Gratacos, ${ }^{4} \mathrm{U}$ Åden. ${ }^{1}$ Department of Women's and Children's Health, Karolinska Institutet, Stockholm, Sweden; ${ }^{2}$ Department of Clinical Neuroscience, Karolinska Institutet, Stockholm, Sweden; ${ }^{3}$ Neuroscience Institute Department of Neurology, Hospital Clinic, Barcelona, Spain; ${ }^{4}$ Department of Women's and Children's Health and Department of Neonatology, Karolinska Institutet, Stockholm, Sweden; ${ }^{5}$ Department of Maternal-Fetal Medicine and Neonatology (ICGON), Hospital Clinic, Barcelona, Spain

\subsection{6/archdischild-2014-307384.1477}

Background and aims Prematurity and intrauterine growth restriction (IUGR) is associated with deviations of the developmental trajectory of the brain. We aimed to examine resting state networks (RSNs) in preterm infants with and without IUGR during natural sleep at 12 months.

Methods We included 30 preterm infants ( $<34$ weeks) without focal brain lesions (12 with IUGR and 18 appropriate for gestational age) and 20 born-term infants that were scanned at 12 months during natural sleep. Structural and functional MRI was acquired in a $3 \mathrm{~T}$ scanner. To account for head movement we performed frame censoring of the data. RSNs were computed using the MELODIC module (FSL software). Dual regression analysis was used to query between-group differences in RSNs.

Results Overall, the degree of movement on functional data was small. In the group we identified nine RSNs encompassing bilaterally the primary visual cortex, auditory cortex, sensori-motor cortex, lateral parietal cortex, precuneus, frontal and a sub-cortical network. Preterm infants had a more prominent cerebellar network compared to term infants. The three groups showed a fragmentized default-mode network. No significant differences were found between groups.

Conclusions The spatial patterns of the RSNs observed in preterm and term infants corresponded closely to those observed in adults. These findings may suggest that IUGR and prematurity does not interfere with the normal process of functional brain network development at 12 months of age. The fact that we could not find differences in RSNs does not rule out that alterations could occur later in development.

\section{PO-0851 SLEEP ISSUES FOR CHILDREN WITH CEREBRAL PALSY: WHY ARE CHILDREN AND THEIR PARENTS AWAKE AT NIGHT? A PILOT STUDY}

${ }^{1} \mathrm{~S}$ Petersen, ${ }^{2} \mathrm{D}$ Reddihough, ${ }^{3} \mathrm{~F}$ Newall. 'Developmental Medicine, Royal Children's Hospital, Melbourne, Australia; ${ }^{2}$ Developmental Disability and Rehabilitation Research, Murdoch Childrens Research Institute, Melbourne, Australia; ${ }^{3}$ Nursing Research, Royal Children's Hospital, Melbourne, Australia

10.1136/archdischild-2014-307384.1478
Background and aims Children with cerebral palsy (CP) and their caregivers often report poor quality sleep. A proportion of sleep problems can be linked to physical care needs associated with CP; need for repositioning, pain and hygiene. Simple but effective changes can be made to care regimes that may reduce sleep disturbance. However, sleep issues and the subsequent sleep solutions are frequently overlooked. The aim of this pilot study is to identify why children with $\mathrm{CP}$ and their parents/caregivers are awake at night-time.

Methods Parents of children with severe CP aged between 6 and 10 years were asked to complete a Time Use Diary (TUD) of their child's night from 6 pm until 9 am for two nights.

Results Eight families returned the study documents, yielding a total of 16 nights of TUD data. Two children slept uninterrupted for both nights. Two children experienced 11 awakenings over the two nights. The remaining 4 children woke $0-2$ times across both nights. Waking time ranged from 15 min to 4 h. Reasons for children waking included; need for repositioning or comforting, distress, pain, restlessness.

Conclusion Children with $\mathrm{CP}$ and their parents/caregivers wake often and for long periods overnight. The TUD provided rich data in regards to night time awakenings for children with $\mathrm{CP}$ and their parents. This data can be used to design sleep interventions to address problematic sleep for this cohort. This study needs to be refined and repeated on a larger scale in order to gain greater breadth of information and validity.

\section{PO-0852 WITHDRAWN}

\section{PO-0853 QUALITY OF LIFE IN CHILDREN WITH CEREBRAL PALSY AND ADHD BEFORE AND AFTER ADMINISTRATION OF TREATMENT}

${ }^{1}$ DA Plesca, 'S Houssein Toufic, ${ }^{1}$ I Dinu, ${ }^{2} \mathrm{M}$ Safer. ${ }^{1}$ Pediatric and Pediatric Neurology Children Clinical Hospital "Victor Gomoiu", University of Medicine and Pharmacy "Carol Davila", Bucharest, Romania; 'Pediatric and Pediatric Neurology Children Clinical Hospital "Victor Gomoiu", "Victor Gomoiu" Children Clinical Hospital, Bucharest, Romania

\subsection{6/archdischild-2014-307384.1479}

Background and aims Attention deficit hyperactivity disorder (ADHD) and cerebral palsy (CP) are chronic diseases with major impact on quality of life (QoL) of children and their families. The goal of the study was to analyse the influence of pharmacologic treatment concerning the expression of satisfaction as key concept in the areas of life, compared to overall QoL and overall satisfaction on QoL.

Methods The scores assigned to the satisfaction in each area of life were statistically analysed with Wilcoxon test. For correlation analysis of the satisfaction in each area of life with overall QoL and overall satisfaction on QoL, non-parametric Spearman test was applied. Family Quality of Life Survey (FQOLS) - the version addressed to the main caregiver of the child with disability was used.

Results Wilcoxon statistic Z, applied to the comparison between the two time points of the scores for satisfaction in each area of life, resulted in associated statistical significance $(p<0.01)$ for all the areas for both $\mathrm{CP}$ and ADHD groups, for the difference between the two time points - before and after treatment.

Conclusions Pharmacologic treatment intervention influenced by a significant improvement the satisfaction in all area of life 